\title{
Tandem addition-cyclization reaction catalyzed by ytterbium chloride: An efficient one-step synthesis of 2-amino-4H-3,1-benzothiazine
}

\author{
HUANG Jie, YU Yong, HUA Lu, YAO ZhiGang, XU Fan* \& SHEN Qi* \\ Key Laboratory of Organic Synthesis, College of Chemistry, Chemical Engineering and Material Science, Soochow University, Suzhou 215123, \\ China
}

Received April 11, 2012; accepted May 28, 2012; published online January 9, 2013

\begin{abstract}
A clean, efficient method to synthesize 2-amino-4H-3,1-benzothiazines by ytterbium chloride-catalyzed tandem addition-cyclization reaction of $o$-aminocinnamate and isothiocyanates under solvent-free conditions is developed.
\end{abstract}

2-amino-3,1-benzothiazine, ytterbium chloride, catalysis, tandem reaction

Citation: Huang J, Yu Y, Hua L, et al. Tandem addition-cyclization reaction catalyzed by ytterbium chloride: An efficient one-step synthesis of 2-amino-4H3,1-benzothiazine. Chin Sci Bull, 2013, 58: 717-723, doi: 10.1007/s11434-012-5631-z

3,1-Benzothiazine skeleton is present in many natural and synthetic biologically active materials. While 3,1-benzothiazines are well studied [1-9] and widely applied in pharmaceutical and biochemical fields [5,10-13], some of their functionalized derivatives such as 2-amino-4H-3,1benzothiazines also possess interesting biological properties [14-17] and have attracted increasing attention. For example, it was recently found that 2-(4-piperidinamino)-4H-3,1benzothiazines (Figure 1, A) show considerable cytoprotective properties toward the heart or neurons [16]. More recently, amidine, thiourea and guanidine derivatives of 2-amino-3,1-benzothiazine (Figure 1, B) were used as novel pharmacological agents for the treatment of neurodegenerative pathologies such as cerebral ischemia, neurodegeneration induced by cranial trauma, and Alzheimer's disease [17]. Encouraged by the potential applications of 2-amino4H-3,1-benzothiazines, some research groups dedicated their effort to developing efficient methods for the synthesis of this type of compound. As a consequence, several approaches to prepare 2-amino-4H-3,1-benzothiazines have been reported [18-31], and most of which are based on the reactions of aromatic amines or thioureas bearing a halomethyl or hydroxymethyl substituent in the ortho position

*Corresponding authors (email: xufan@ suda.edu.cn; qshen@suda.edu.cn)



A

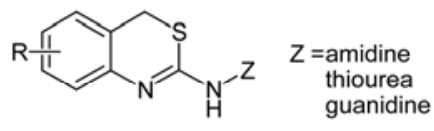

B
Figure 1 Potential pharmacological agents containing a 2-amino-3,1benzothiazine core.

of the aromatic ring. We noticed that a strong Bronsted acid [18-22] or noble metal catalyst [23,24] was required for a smooth reaction in most cases. At the same time, heating under reflux in organic solvents, complex and inaccessible substrates, and multistep reaction processes appeared to be essential conditions to obtain satisfactory results. Therefore, further development of novel catalysts and relevant processes of high efficiency to synthesize 2-amino- $4 H-3,1-$ benzothiazine in a simple and clean manner remains of great interest.

Over the past decades, notable progress has been made in the use of lanthanide reagents as catalysts in organic synthesis. Lanthanides are nontoxic and relatively abundant in nature, and the success in developing many useful reactions efficiently catalyzed by lanthanide compounds is attributed 
to the unique features of lanthanide centers such as high electrophilicity, variable metal ion radius and tunable coordination patterns. As a continuation of our interest in the applications of lanthanide-catalyzed carbon-nitrogen and carbon-phosphorus bond-forming reactions [32-40] for the construction of heterocycles, we investigated the effectiveness of lanthanide chlorides as catalysts for carbon-sulfur bond-forming reactions. Herein, a highly efficient process affording 2-amino-4H-3,1-benzothiazines by lanthanide chloride-catalyzed tandem addition-cyclization reaction of $o$-aminocinnamate and isothiocyanates is presented.

\section{Experimental}

\subsection{General remarks}

Lanthanide chlorides were synthesized according to the method described by Taylor and Carter [41]. Melting points were uncorrected. ${ }^{1} \mathrm{H}$ and ${ }^{13} \mathrm{C}$ NMR spectra were obtained on Varian INOVA-400 and System-300 spectrometers using tetramethylsilane (TMS) as an internal reference. IR spectra were obtained on a Nicolet FT-IR 1000 spectrophotometer. HRMS data were recorded on a Micromass GCT instrument.

\subsection{General procedure}

A mixture of ethyl $o$-aminocinnamate $(1 \mathrm{mmol})$, isothiocyanate $(1.2 \mathrm{mmol})$ and ytterbium trichloride $(0.025 \mathrm{mmol})$ was stirred at the desired temperature for the given time. Water was added, and the mixture was extracted with EtOAc. The combined organic layers were dried with anhydrous $\mathrm{Na}_{2} \mathrm{SO}_{4}$, concentrated in vacuo, and purified by chromatography on silica gel [eluent: EtOAc/petroleum ether (60$\left.\left.90^{\circ} \mathrm{C}\right) 1: 10\right]$ to afford the desired 2-amino-4H-3,1-benzothiazine.

Ethyl 2-(2-(phenylamino)-4H-benzo[ $d][1,3]$ thiazin-4-yl) acetate (3a). $\operatorname{mp~} 113-114^{\circ} \mathrm{C} ;{ }^{1} \mathrm{H} \mathrm{NMR}\left(400 \mathrm{MHz}^{-} \mathrm{CDCl}_{3}\right)$ $\delta 7.57(\mathrm{~d}, J=8.0 \mathrm{~Hz}, 2 \mathrm{H}), 7.36-7.27(\mathrm{~m}, 3 \mathrm{H}), 7.22-7.16(\mathrm{~m}$, $2 \mathrm{H}), 7.09(\mathrm{t}, J=7.2 \mathrm{~Hz}, 2 \mathrm{H}), 6.71$ (br s, $1 \mathrm{H}), 4.51$ (dd, $J=8.8$, $6.4 \mathrm{~Hz}, 1 \mathrm{H}), 4.14$ (q, $J=7.2 \mathrm{~Hz}, 2 \mathrm{H}), 2.83$ (dd, $J=16.0,8.8$ $\mathrm{Hz}, 1 \mathrm{H}), 2.75$ (dd, $J=16.0,6.4 \mathrm{~Hz}, 1 \mathrm{H}), 1.22$ (t, $J=7.2 \mathrm{~Hz}$, $3 \mathrm{H}) ;{ }^{13} \mathrm{C}$ NMR $\left(75 \mathrm{MHz}, \mathrm{CDCl}_{3}\right) \delta 170.7,149.6,143.1$, $141.8,129.4,129.1,126.9,124.5,124.3,124.0,122.8$, 121.2, 61.3, 42.0, 40.7, 14.6; IR (KBr) $v$ 691, 757, 1494, 1576, 1732, $2973 \mathrm{~cm}^{-1}$; HRMS calcd. for $\mathrm{C}_{18} \mathrm{H}_{18} \mathrm{~N}_{2} \mathrm{O}_{2} \mathrm{~S}$ 326.1089 , found 326.1095 .

Ethyl 2-(2-(phenylimino)-2,4-dihydro- $1 H$-benzo[d][1,3] thiazin-4-yl)acetate (4a). ${ }^{1} \mathrm{H}$ NMR (400 MHz, DMSO-d 6 ) $\delta$ 9.54 (br s, 1H), 7.97-7.79 (m, 2H), 7.33-7.21 (m, 4H), 7.13$6.99(\mathrm{~m}, 3 \mathrm{H}), 4.59$ (t, $J=7.6 \mathrm{~Hz}, 1 \mathrm{H}), 4.07$ (q, $J=7.2 \mathrm{~Hz}, 2 \mathrm{H})$, $2.75(\mathrm{dd}, J=16.0,6.4 \mathrm{~Hz}, 1 \mathrm{H}), 2.68$ (dd, $J=16.0,9.6 \mathrm{~Hz}, 1 \mathrm{H})$, $1.16(\mathrm{t}, J=7.2 \mathrm{~Hz}, 3 \mathrm{H})$.

Ethyl 2-(2-(4-fluorophenylamino)-4H-benzo[d][1,3]thiazin- 4-yl)acetate (3b). mp $112-114{ }^{\circ} \mathrm{C} ;{ }^{1} \mathrm{H}$ NMR $(400 \mathrm{MHz}$, $\left.\mathrm{CDCl}_{3}\right) \delta 7.43-7.40(\mathrm{~m}, 2 \mathrm{H}), 7.24(\mathrm{~d}, J=7.6 \mathrm{~Hz}, 1 \mathrm{H}), 7.16$ (d, J=7.2 Hz, 1H), 7.10-7.00 (m, 4H), 6.40 (br s, 1H), 4.50 (t, $J=7.6 \mathrm{~Hz}, 1 \mathrm{H}), 4.13$ (q, $J=7.2 \mathrm{~Hz}, 2 \mathrm{H}), 2.84$ (dd, $J=16.0$, $8.8 \mathrm{~Hz}, 1 \mathrm{H}$ ), 2.76 (dd, $J=16.0,6.4 \mathrm{~Hz}, 1 \mathrm{H}$ ), 1.22 (t, $J=7.2$ $\mathrm{Hz}, 3 \mathrm{H}) ;{ }^{13} \mathrm{C}$ NMR $\left(75 \mathrm{MHz}, \mathrm{CDCl}_{3}\right) \delta 170.2,160.7,157.5$, $150.5,141.7,138.5,128.6,126.4,123.8,122.7,115.6$, 115.3, 60.9, 41.5, 40.2, 14.1; IR (KBr) v 752, 837, 1149, 1226, 1300, 1439, 1504, 1581, 1736, $2985 \mathrm{~cm}^{-1}$; HRMS calcd. for $\mathrm{C}_{18} \mathrm{H}_{17} \mathrm{FN}_{2} \mathrm{O}_{2} \mathrm{~S} 344.0995$, found 344.0995.

Ethyl 2-(2-(4-chlorophenylimino)-2,4-dihydro- $1 H$-benzo [d][1,3]thiazin-4-yl)acetate (4c). mp $130-132^{\circ} \mathrm{C} ;{ }^{1} \mathrm{H}$ NMR (400 MHz, DMSO-d d $_{6} \delta 9.72$ (br s, 1H), $7.96(\mathrm{~m}, 2 \mathrm{H}), 7.37$ $(\mathrm{d}, J=8.4 \mathrm{~Hz}, 2 \mathrm{H}), 7.31-7.23(\mathrm{~m}, 2 \mathrm{H}), 7.16-7.07(\mathrm{~m}, 2 \mathrm{H})$, $4.63(\mathrm{t}, J=7.6 \mathrm{~Hz}, 1 \mathrm{H}), 4.07(\mathrm{q}, J=7.2 \mathrm{~Hz}, 2 \mathrm{H}), 2.76$ (dd, $J=$ 16.0, $6.0 \mathrm{~Hz}, 1 \mathrm{H}), 2.69$ (dd, $J=16.0,8.8 \mathrm{~Hz}, 1 \mathrm{H}), 1.17$ (t, $J=$ $7.2 \mathrm{~Hz}, 3 \mathrm{H}) ;{ }^{13} \mathrm{C}$ NMR $\left(75 \mathrm{MHz}, \mathrm{CDCl}_{3}\right) \delta 170.7,149.9$, $142.4,141.1,129.4,129.2,128.9,127.0,124.7,123.7$, 122.7, 122.5, 61.4, 42.0, 40.7, 14.6; IR (KBr) v 741, 844, 1092, 1337, 1485, 1575, 1730, $2911 \mathrm{~cm}^{-1}$; HRMS calcd. for $\mathrm{C}_{18} \mathrm{H}_{17} \mathrm{ClN}_{2} \mathrm{O}_{2} \mathrm{~S} 360.0699$, found 360.0698.

Ethyl 2-(2-(4-bromophenylamino)-4H-benzo[ $d][1,3]$ thiazin4-yl)acetate (3d). mp $130-132{ }^{\circ} \mathrm{C} ;{ }^{1} \mathrm{H}$ NMR (400 MHz, $\left.\mathrm{CDCl}_{3}\right) \delta 7.45-7.40(\mathrm{~m}, 4 \mathrm{H}), 7.30-7.28(\mathrm{~m}, 1 \mathrm{H}), 7.17-7.07$ (m, 3H), 6.95 (br s, 1H), 4.51 (dd, J=8.8, 6.4 Hz, 1H), 4.14 (q, $J=7.2 \mathrm{~Hz}, 2 \mathrm{H}), 2.83$ (dd, $J=16.0,8.8 \mathrm{~Hz}, 1 \mathrm{H}), 2.75$ (dd, $J=16.0,6.4 \mathrm{~Hz}, 1 \mathrm{H}), 1.22(\mathrm{t}, J=7.2 \mathrm{~Hz}, 3 \mathrm{H}) ;{ }^{13} \mathrm{C}$ NMR $(75$ $\left.\mathrm{MHz}, \mathrm{CDCl}_{3}\right) \delta 170.7,150.0,142.3,141.7,132.3,129.2$, 127.0, 124.6, 123.6, 122.9, 122.7, 116.6, 61.5, 42.0, 40.7, 14.6; IR (KBr) $v 742,841,1337,1562,1728,2926 \mathrm{~cm}^{-1}$; HRMS calcd. for $\mathrm{C}_{18} \mathrm{H}_{17} \mathrm{BrN}_{2} \mathrm{O}_{2} \mathrm{~S}$ 404.0194, found 404.0200.

Ethyl 2-(2-(4-nitrophenylamino)-4H-benzo[d][1,3]thiazin4-yl)acetate (3e). mp $156-156.5^{\circ} \mathrm{C}$; ${ }^{1} \mathrm{H}$ NMR (400 MHz, $\left.\mathrm{CDCl}_{3}\right) \delta 8.14(\mathrm{~d}, J=9.2 \mathrm{~Hz}, 2 \mathrm{H}), 7.63(\mathrm{~d}, J=8.4 \mathrm{~Hz}, 2 \mathrm{H})$, $7.27-7.23(\mathrm{~m}, 1 \mathrm{H}), 7.15-7.05(\mathrm{~m}, 3 \mathrm{H}), 4.48(\mathrm{dd}, J=8.8,6.8$ $\mathrm{Hz}, 1 \mathrm{H}), 4.08$ (q, J=7.2 Hz, 2H), 2.77 (dd, $J=16.0,8.8 \mathrm{~Hz}$, $1 \mathrm{H}), 2.68(\mathrm{dd}, J=16.4,6.4 \mathrm{~Hz}, 1 \mathrm{H}), 1.16(\mathrm{t}, J=7.2 \mathrm{~Hz}, 3 \mathrm{H})$; ${ }^{13} \mathrm{C}$ NMR $\left(75 \mathrm{MHz}, \mathrm{CDCl}_{3}\right) \delta 170.0,148.6,147.7,142.6$, $141.5,128.9,126.6,125.4,125.0,123.8,122.0,119.7,61.1$, 41.6, 40.2, 14.1; IR (KBr) v 749, 848, 1226, 1304, 1331, 1407, 1449, 1502, 1558, 1582, 1719, $2983 \mathrm{~cm}^{-1}$; HRMS calcd. for $\mathrm{C}_{18} \mathrm{H}_{17} \mathrm{~N}_{3} \mathrm{O}_{4} \mathrm{~S} 371.0940$, found 371.0943 .

Ethyl 2-(2-(4-nitrophenylimino)-2,4-dihydro- $1 H$-benzo $[d]$ $[1,3]$ thiazin-4-yl)acetate (4e). ${ }^{1} \mathrm{H}$ NMR (400 MHz, DMSO$\left.\mathrm{d}_{6}\right) \delta 10.25($ br s, $1 \mathrm{H}), 8.24-8.15(\mathrm{~m}, 4 \mathrm{H}), 7.35-7.14(\mathrm{~m}$, $4 \mathrm{H}), 4.68$ (dd, $J=8.8,6.4 \mathrm{~Hz}, 1 \mathrm{H}), 4.07$ (q, $J=7.2 \mathrm{~Hz}, 2 \mathrm{H})$, 2.77 (dd, $J=16.0,6.4 \mathrm{~Hz}, 1 \mathrm{H}), 2.70$ (dd, $J=16.0,8.8 \mathrm{~Hz}, 1 \mathrm{H})$, $1.17(\mathrm{t}, J=7.2 \mathrm{~Hz}, 3 \mathrm{H})$.

Ethyl 2-(2-(3-chlorophenylamino)-4H-benzo[ $d][1,3]$ thiazin4-yl)acetate (3f). mp $107-109^{\circ} \mathrm{C} ;{ }^{1} \mathrm{H}$ NMR $(400 \mathrm{MHz}$, $\left.\mathrm{CDCl}_{3}\right) \delta 7.74-7.69(\mathrm{~m}, 1 \mathrm{H}), 7.33-7.29(\mathrm{~m}, 2 \mathrm{H}), 7.24-7.04$ $(\mathrm{m}, 5 \mathrm{H}), 6.76$ (br s, 1H), 4.52 (dd, $J=8.8,6.8 \mathrm{~Hz}, 1 \mathrm{H}), 4.15$ (q, $J=7.2 \mathrm{~Hz}, 2 \mathrm{H}), 2.83(\mathrm{dd}, J=16.0,8.8 \mathrm{~Hz}, 1 \mathrm{H}), 2.75$ (dd, $J=16.0,6.4 \mathrm{~Hz}, 1 \mathrm{H}), 1.23(\mathrm{t}, J=7.2 \mathrm{~Hz}, 3 \mathrm{H}) ;{ }^{13} \mathrm{C} \mathrm{NMR}$ 
$\left(75 \mathrm{MHz}, \mathrm{CDCl}_{3}\right) \delta 170.7,150.3,144.1,142.0,134.9,130.3$, 129.2, 127.0, 124.6, 124.0, 123.4, 122.7, 121.4, 119.4, 61.5, 42.0, 40.7, 14.6; IR (KBr) v700, 756, 779, 879, 1043, 1240, 1307, 1439, 1466, 1499, 1574, 1721, $2980 \mathrm{~cm}^{-1}$; HRMS calcd. for $\mathrm{C}_{18} \mathrm{H}_{17} \mathrm{ClN}_{2} \mathrm{O}_{2} \mathrm{~S} 360.0699$, found 360.0706.

Ethyl 2-(2-(3-chlorophenylimino)-2,4-dihydro-1H-benzo [d][1,3]thiazin-4-yl)acetate (4f). ${ }^{1} \mathrm{H}$ NMR (400 MHz, DMSO$\left.\mathrm{d}_{6}\right) \delta 9.74($ br s, $1 \mathrm{H}), 8.17-7.05(\mathrm{~m}, 8 \mathrm{H}), 4.62(\mathrm{t}, J=7.2 \mathrm{~Hz}$, $1 \mathrm{H}), 4.07$ (q, $J=7.2 \mathrm{~Hz}, 2 \mathrm{H}), 2.75$ (dd, $J=15.6,6.0 \mathrm{~Hz}, 1 \mathrm{H})$, 2.67 (dd, $J=15.6,8.8 \mathrm{~Hz}, 1 \mathrm{H}), 1.16(\mathrm{t}, J=6.8 \mathrm{~Hz}, 3 \mathrm{H})$.

Ethyl 2-(2-(3-methoxyphenylamino)-4H-benzo[d][1,3]thiazin-4-yl)acetate (3g). mp $87-89^{\circ} \mathrm{C} ;{ }^{1} \mathrm{H}$ NMR $(400 \mathrm{MHz}$, $\left.\mathrm{CDCl}_{3}\right) \delta 7.38(\mathrm{~s}, 1 \mathrm{H}), 7.28-6.94(\mathrm{~m}, 6 \mathrm{H}), 6.64(\mathrm{~d}, J=8.0 \mathrm{~Hz}$, $1 \mathrm{H}), 4.50(\mathrm{t}, J=7.6 \mathrm{~Hz}, 1 \mathrm{H}), 4.13(\mathrm{q}, J=7.2 \mathrm{~Hz}, 2 \mathrm{H}), 3.82(\mathrm{~s}$, $3 \mathrm{H}), 2.84(\mathrm{dd}, J=16.0,8.4 \mathrm{~Hz}, 1 \mathrm{H}), 2.75$ (dd, $J=16.0,6.8 \mathrm{~Hz}$, $1 \mathrm{H}), 1.22(\mathrm{t}, J=7.2 \mathrm{~Hz}, 3 \mathrm{H}) ;{ }^{13} \mathrm{C}$ NMR $\left(75 \mathrm{MHz}, \mathrm{CDCl}_{3}\right) \delta$ $170.1,159.8,150.0,143.2,141.8,129.3,128.4,126.3$, $123.7,123.0,122.2,113.2,109.3,106.6,60.7,55.0,41.4$, 40.0, 14.0; IR (KBr) v 748, 775, 864, 1049, 1192, 1234, 1303, 1435, 1496, 1581, 1727, $2962 \mathrm{~cm}^{-1}$; HRMS calcd. for $\mathrm{C}_{19} \mathrm{H}_{20} \mathrm{~N}_{2} \mathrm{O}_{3} \mathrm{~S} 356.1195$, found 356.1189 .

Ethyl 2-(2-(3-methoxyphenylimino)-2,4-dihydro-1H-benzo [d][1,3]thiazin-4-yl)acetate (4g). ${ }^{1} \mathrm{H}$ NMR (400 MHz, DMSO$\left.\mathrm{d}_{6}\right) \delta 9.56($ br s, $1 \mathrm{H}), 7.79-7.25(\mathrm{~m}, 3 \mathrm{H}), 7.23-7.17(\mathrm{~m}, 2 \mathrm{H})$, 7.13-7.07 (m, 2H), 6.60-6.58 (m, 1H), $4.60(\mathrm{t}, J=7.6 \mathrm{~Hz}$, $1 \mathrm{H}), 4.06$ (q, $J=7.2 \mathrm{~Hz}, 2 \mathrm{H}), 3.76(\mathrm{~s}, 3 \mathrm{H}), 2.75$ (dd, $J=15.6$, $6.4 \mathrm{~Hz}, 1 \mathrm{H}), 2.67(\mathrm{dd}, J=15.6,8.8 \mathrm{~Hz}, 1 \mathrm{H}), 1.16(\mathrm{t}, J=7.2$ $\mathrm{Hz}, 3 \mathrm{H})$.

Ethyl 2-(2-(p-toluidino)-4H-benzo[ $d][1,3]$ thiazin-4-yl)acetate (3h). mp $116-119^{\circ} \mathrm{C} ;{ }^{1} \mathrm{H}$ NMR $\left(400 \mathrm{MHz}, \mathrm{CDCl}_{3}\right) \delta$ $7.43(\mathrm{~d}, J=8.0 \mathrm{~Hz}, 2 \mathrm{H}), 7.30-7.28(\mathrm{~m}, 1 \mathrm{H}), 7.20-7.05(\mathrm{~m}$, $5 \mathrm{H}), 6.66$ (br s, 1H), 4.50 (t, $J=7.6 \mathrm{~Hz}, 1 \mathrm{H}), 4.13$ (q, $J=7.2$ $\mathrm{Hz}, 2 \mathrm{H}), 2.82$ (dd, $J=16.0,8.4 \mathrm{~Hz}, 1 \mathrm{H}), 2.74$ (dd, $J=16.0$, $6.4 \mathrm{~Hz}, 1 \mathrm{H}), 2.33(\mathrm{~s}, 3 \mathrm{H}), 1.22(\mathrm{t}, J=7.2 \mathrm{~Hz}, 3 \mathrm{H}) ;{ }^{13} \mathrm{C} \mathrm{NMR}$ $\left(75 \mathrm{MHz}, \mathrm{CDCl}_{3}\right) \delta 170.8,150.0,143.1,139.4,133.7,129.8$, $129.0,126.9,124.2,124.0,122.8,121.5,61.3,42.0,40.7$, 21.3, 14.6; IR (KBr) $v 748,833,1244,1300,1438,1465$, 1504, 1581, 1730, $2968 \mathrm{~cm}^{-1}$; HRMS calcd. for $\mathrm{C}_{19} \mathrm{H}_{20^{-}}$ $\mathrm{N}_{2} \mathrm{O}_{2} \mathrm{~S} 340.1245$, found 340.1245.

Ethyl 2-(2-(4-methoxyphenylamino)-4H-benzo[d][1,3]thiazin-4-yl)acetate (3i). mp $107-109{ }^{\circ} \mathrm{C} ;{ }^{1} \mathrm{H}$ NMR (400 MHz, $\left.\mathrm{CDCl}_{3}\right) \delta 7.41(\mathrm{~d}, J=8.4 \mathrm{~Hz}, 2 \mathrm{H}), 7.28-7.24(\mathrm{~m}, 1 \mathrm{H}), 7.16-$ $7.04(\mathrm{~m}, 3 \mathrm{H}), 6.88$ (d, J=8.8 Hz, 2H), 4.49 (dd, $J=8.0,6.8$ $\mathrm{Hz}, 1 \mathrm{H}), 4.13$ (q, J=7.2 Hz, 2H), $3.81(\mathrm{~s}, 3 \mathrm{H}), 2.83$ (dd, $J=$ 16.0, $8.4 \mathrm{~Hz}, 1 \mathrm{H}), 2.75$ (dd, $J=16.0,6.8 \mathrm{~Hz}, 1 \mathrm{H}), 1.22$ (t, $J=$ $7.2 \mathrm{~Hz}, 3 \mathrm{H}) ;{ }^{13} \mathrm{C} \mathrm{NMR}\left(75 \mathrm{MHz}, \mathrm{CDCl}_{3}\right) \delta 170.8,156.6$, $150.7,143.1,135.0,129.0,126.9,124.1,123.9,123.5$, $122.8,114.5,61.3,55.9,42.0,40.7,14.6$; IR (KBr) $v 752$, 829, 1028, 1199, 1240, 1298, 1441, 1504, 1581, 1734, 2984 $\mathrm{cm}^{-1}$; HRMS calcd. for $\mathrm{C}_{19} \mathrm{H}_{20} \mathrm{~N}_{2} \mathrm{O}_{3} \mathrm{~S}$ 356.1195, found 356.1199 .

Ethyl 2-(2-(4-methoxyphenylimino)-2,4-dihydro-1H-benzo $[d][1,3]$ thiazin-4-yl)acetate (4i). ${ }^{1} \mathrm{H}$ NMR (400 MHz, DMSO$\left.\mathrm{d}_{6}\right) \delta 9.43($ br s, $1 \mathrm{H}), 7.78(\mathrm{~m}, 2 \mathrm{H}), 7.27-7.20(\mathrm{~m}, 2 \mathrm{H})$,
7.09-7.02 (m, 2H), 6.90 (d, J=8.8 Hz, 2H), 4.58 (dd, $J=8.8$, $6.8 \mathrm{~Hz}, 1 \mathrm{H}), 4.07$ (q, J=7.2 Hz, 2H), 3.74 (s, 3H), 2.75 (dd, $J=15.6,6.8 \mathrm{~Hz}, 1 \mathrm{H}), 2.67(\mathrm{dd}, J=15.6,8.8 \mathrm{~Hz}, 1 \mathrm{H}), 1.17(\mathrm{t}$, $J=7.2 \mathrm{~Hz}, 3 \mathrm{H})$.

Ethyl 2-(2-(2-fluorophenylamino)-4H-benzo[ $d][1,3]$ thiazin4-yl)acetate (3j). mp $127-128.5^{\circ} \mathrm{C}$; ${ }^{1} \mathrm{H}$ NMR (400 MHz, $\left.\mathrm{CDCl}_{3}\right) \delta 8.37$ (br s, 1H), 7.31-6.99 (m, 8H), 4.52 (dd, $J=$ 8.4, $6.8 \mathrm{~Hz}, 1 \mathrm{H}), 4.15$ (q, J=7.2 Hz, 2H), 2.85 (dd, $J=16.4$, $8.8 \mathrm{~Hz}, 1 \mathrm{H}), 2.76(\mathrm{dd}, J=16.0,6.4 \mathrm{~Hz}, 1 \mathrm{H}), 1.23(\mathrm{t}, J=7.2$ $\mathrm{Hz}, 3 \mathrm{H}) ;{ }^{13} \mathrm{C}$ NMR $\left(75 \mathrm{MHz}, \mathrm{CDCl}_{3}\right) \delta 170.2,154.4,151.2$, $149.7,149.2,141.9,128.6,126.5,124.3,123.6,122.5$, $122.3,115.1,114.8,60.9,41.4,40.2$, 14.1; IR (KBr) $v 752$, 775, 1226, 1249, 1300, 1435, 1497, 1581, 1724, $2977 \mathrm{~cm}^{-1}$; HRMS calcd. for $\mathrm{C}_{18} \mathrm{H}_{17} \mathrm{FN}_{2} \mathrm{O}_{2} \mathrm{~S} 344.0995$, found 344.0997.

Ethyl 2-(2-(2-chlorophenylamino)-4H-benzo[ $d][1,3]$ thiazin4-yl)acetate (3k). mp $132-132.5^{\circ} \mathrm{C}$; ${ }^{1} \mathrm{H}$ NMR $(300 \mathrm{MHz}$, $\left.\mathrm{CDCl}_{3}\right) \delta 8.42$ (br s, $\left.1 \mathrm{H}\right), 7.40-7.28(\mathrm{~m}, 3 \mathrm{H}), 7.21-6.98(\mathrm{~m}$, $5 \mathrm{H}), 4.53(\mathrm{dd}, J=8.7,6.9 \mathrm{~Hz}, 1 \mathrm{H}), 4.20-4.09$ (m, $J=7.2 \mathrm{~Hz}$, 2H), 2.86 (dd, $J=16.2,8.7 \mathrm{~Hz}, 1 \mathrm{H}), 2.76$ (dd, $J=15.6,6.6 \mathrm{~Hz}$, $1 \mathrm{H}), 1.23(\mathrm{t}, J=7.2 \mathrm{~Hz}, 3 \mathrm{H}) ;{ }^{13} \mathrm{C}$ NMR $\left(75 \mathrm{MHz}, \mathrm{CDCl}_{3}\right) \delta$ $170.6,150.8,141.8,140.2,129.7,129.1,127.9,127.0$, $124.9,124.6,124.5,123.3,123.0,122.8,61.3,41.9,40.6$, 14.6; IR (KBr) v 749, 1035, 1240, 1340, 1496, 1574, 1724, $2921 \mathrm{~cm}^{-1}$; HRMS calcd. for $\mathrm{C}_{18} \mathrm{H}_{17} \mathrm{ClN}_{2} \mathrm{O}_{2} \mathrm{~S} 360.0699$, found 360.0694 .

Ethyl 2-(2-(cyclohexylamino)-4H-benzo[d][1,3]thiazin-4yl)acetate (3l). mp 93.5-95.5 ${ }^{\circ} \mathrm{C} ;{ }^{1} \mathrm{H}$ NMR (400 MHz, $\left.\mathrm{CDCl}_{3}\right) \delta 7.25-6.99(\mathrm{~m}, 4 \mathrm{H}), 4.59$ (br s, $\left.1 \mathrm{H}\right), 4.43$ (dd, $J=$ 8.4, $6.8 \mathrm{~Hz}, 1 \mathrm{H}), 4.14$ (q, J=7.2 Hz, 2H), 4.07-3.97 (m, 1H), 2.75 (dd, $J=16.0,8.8 \mathrm{~Hz}, 1 \mathrm{H}), 2.69$ (dd, $J=16.0,6.4 \mathrm{~Hz}, 1 \mathrm{H}$ ), 2.15-1.13 (m, 13H); ${ }^{13} \mathrm{C}$ NMR $\left(75 \mathrm{MHz}, \mathrm{CDCl}_{3}\right) \delta 170.9$, 151.0, 145.3, 128.9, 126.7, 125.4, 123.7, 122.7, 61.2, 51.5, 41.8, 40.6, 34.1, 33.5, 26.1, 25.3, 25.2, 14.6; IR (KBr) v 761, $1220,1488,1574,1728,2936,3391 \mathrm{~cm}^{-1}$; HRMS calcd. for $\mathrm{C}_{18} \mathrm{H}_{24} \mathrm{~N}_{2} \mathrm{O}_{2} \mathrm{~S} 332.1559$, found 332.1550.

Ethyl 2-(2-(cyclohexylimino)-2,4-dihydro-1H-benzo[ $d][1,3]$ thiazin-4-yl)acetate (4l). ${ }^{1} \mathrm{H}$ NMR (400 MHz, DMSO-d 6 ) $\delta$ $7.26(\mathrm{~d}, J=6.4 \mathrm{~Hz}, 1 \mathrm{H}), 7.18-7.11(\mathrm{~m}, 2 \mathrm{H}), 6.94-6.92$ (m, 2H), 4.43 (t, J=7.6 Hz, 1H), 4.05 (q, $J=6.8 \mathrm{~Hz}, 2 \mathrm{H}), 3.97-$ 3.89 (m, 1H), 2.68-2.55 (m, 2H), 1.99-1.11 (m, 13H).

Ethyl 2-(2-(methylamino)-4H-benzo[ $d][1,3]$ thiazin-4-yl) acetate $(3 \mathbf{m})$. mp $138-140^{\circ} \mathrm{C} ;{ }^{1} \mathrm{H}$ NMR $\left(400 \mathrm{MHz}, \mathrm{CDCl}_{3}\right)$ $\delta 7.27-7.24(\mathrm{~m}, 1 \mathrm{H}), 7.17(\mathrm{~d}, J=8.0 \mathrm{~Hz}, 1 \mathrm{H}), 7.12$ (d, $J=7.2$ $\mathrm{Hz}, 1 \mathrm{H}), 7.02$ (t, J=7.2 Hz, 1H), 4.43 (t, J=7.6 Hz, 1H), 4.14 $(\mathrm{q}, J=7.2 \mathrm{~Hz}, 2 \mathrm{H}), 3.09(\mathrm{~s}, 3 \mathrm{H}), 2.75(\mathrm{dd}, J=16.0,8.8 \mathrm{~Hz}$, 1H), 2.68 (dd, $J=16.0,6.8 \mathrm{~Hz}, 1 \mathrm{H}), 1.23$ (t, $J=7.2 \mathrm{~Hz}, 3 \mathrm{H})$; ${ }^{13} \mathrm{C}$ NMR $\left(75 \mathrm{MHz}, \mathrm{CDCl}_{3}\right) \delta 170.4,152.0,144.7,128.5$, $126.3,125.1,123.4,122.3,60.8,41.5,40.1,29.3,14.1$; IR (KBr) $v 761,1221,1411,1450,1478,1604,1730,2889$, $2975 \mathrm{~cm}^{-1}$; HRMS calcd. for $\mathrm{C}_{13} \mathrm{H}_{16} \mathrm{~N}_{2} \mathrm{O}_{2} \mathrm{~S} 264.0932$, found 265.1005 .

Ethyl 2-(2-(methylimino)-2,4-dihydro- $1 H$-benzo[d][1,3] thiazin-4-yl)acetate (4m). ${ }^{1} \mathrm{H}$ NMR (400 MHz, DMSO-d 6 ) $\delta$ 7.33 (br s, 1H), 7.20-7.12 (m, 2H), 6.98-6.92 (m, 2H), 4.45 
(dd, $J=8.8,6.4 \mathrm{~Hz}, 1 \mathrm{H}), 4.05$ (q, $J=7.2 \mathrm{~Hz}, 2 \mathrm{H}), 2.87$ (d, $J=$ $4.0 \mathrm{~Hz}, 3 \mathrm{H}), 2.65(\mathrm{dd}, J=15.6,6.4 \mathrm{~Hz}, 1 \mathrm{H}), 2.56(\mathrm{dd}, J=$ $16.0,8.8 \mathrm{~Hz}, 1 \mathrm{H}), 1.15(\mathrm{t}, J=7.2 \mathrm{~Hz}, 3 \mathrm{H})$.

\section{Results and discussion}

In an initial experiment, ethyl $o$-aminocinnamate (1) was reacted with phenyl isothiocyanate (2a) (Scheme 1) in the presence of $10 \mathrm{~mol} \%$ of ytterbium chloride $\left(\mathrm{YbCl}_{3}\right)$, a readily available and economical lanthanide Lewis acid. After work up, a compound was isolated in quantitative yield (99\%) and high purity as judged by HPLC. The results of X-ray diffraction analysis of a single crystal of this compound [42] (Figure 2) indicated that the product was the expected ethyl 2-(2-(phenylamino)-4H-benzo[d][1,3]thiazin-4-yl)acetate (3a). However, after careful identification, it was found that the average C1-N1 bond distance of $1.363 \AA$ was longer than the $\mathrm{C} 1-\mathrm{N} 2$ bond length of $1.288 \AA$. According to the literature $[43,44]$, the typical length of a C-N bond is $1.339 \AA$, while that of a $\mathrm{C}=\mathrm{N}$ double bond is $1.279 \AA$. Thus, the structure of the product in crystalline state is actually 2-(2(phenylimino)-2,4-dihydro-1H-benzo[ $d][1,3]$ thiazin-4-yl)acetate (4a), in which the $\mathrm{C}=\mathrm{N}$ double bond is exocyclic. To confirm the structure of the product, ${ }^{1} \mathrm{H}$ NMR spectra recorded in different deuterated solvents were studied. The compound produces a signal at $\delta 6.7$ in $\mathrm{CDCl}_{3}$, which is in accord with the chemical shift of the exocyclic $\mathrm{N}-\mathrm{H}$ group $[20,21]$ in 3a. Meanwhile, the signal of the $\mathrm{N}-\mathrm{H}$ group in the ${ }^{1} \mathrm{H}$ NMR spectrum recorded in DMSO-d $\mathrm{d}_{6}$ appears at $\delta 9.5$, correlating with the corresponding value of the proton of the endocyclic N-H group $[31,45,46]$ in 4 a. These findings suggest that the product present in both DMSO- $\mathrm{d}_{6}$ and the single crystal is $\mathbf{4 a}$. When the crystal dissolves in $\mathrm{CDCl}_{3}$, it<smiles>CCOC(=O)/C=C/c1ccccc1N</smiles>

Scheme 1

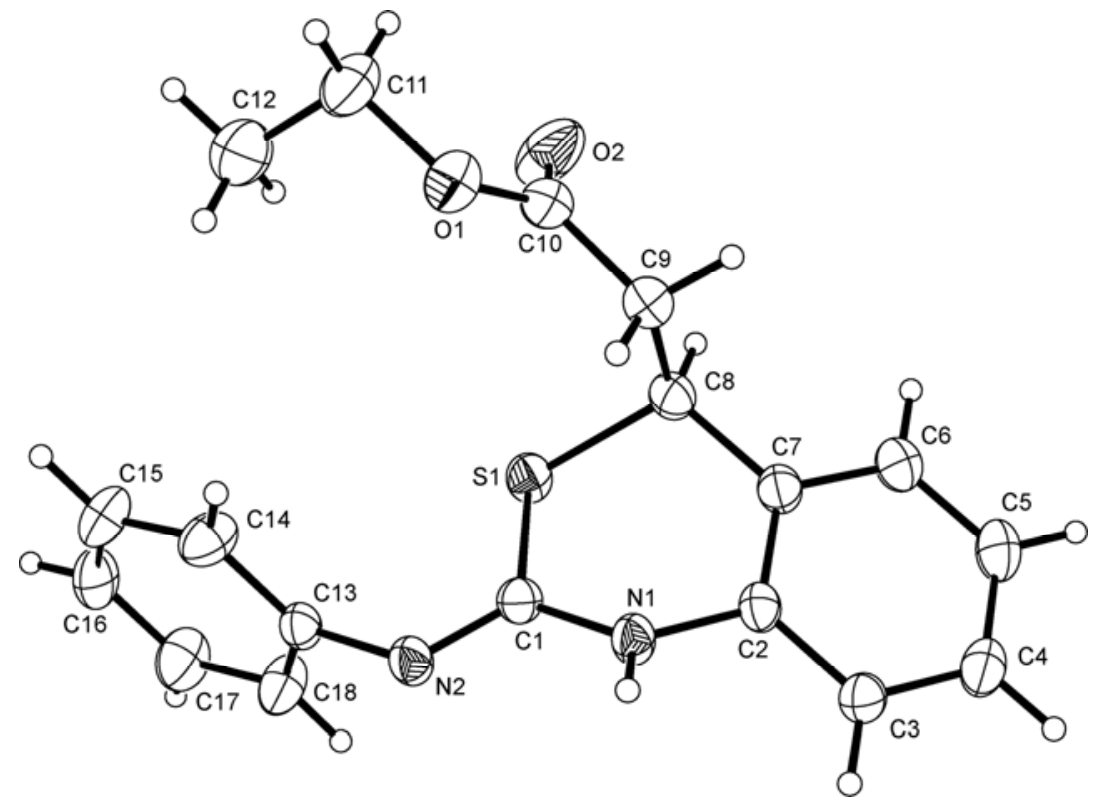

Figure 2 X-ray crystal structure of $\mathbf{4 a}$. 
readily undergoes tautomeric transformation from $\mathbf{4 a}$ into 3a. It can be confirmed that solvent conditions play an important role in affecting the tautomeric equilibrium. This result is slightly different from that obtained by Shabarov and coworkers [22]. Interestingly, another type of cyclization product 5 (Scheme 1), which can be formed through an intramolecular $N$-nucleophilic attack rather than $S$-nucleophilic attack, was not observed.

Various conditions including time, temperature, catalyst loading, and the molar ratio of the respective substrates were then screened to optimize this catalytic system. The results are listed in Table 1 . Use of $2.5 \mathrm{~mol} \%$ of $\mathrm{YbCl}_{3}$ is sufficient to give the product in $99 \%$ yield, and a slight excess of isothiocyanate promotes conversion. Thus, reaction conditions of $2.5 \mathrm{~mol} \%$ catalyst at $50^{\circ} \mathrm{C}$ for $4 \mathrm{~h}$ were used for the following studies.

A series of metal chlorides were used as Lewis acids to assess the influence of the central metal ion on catalytic activity. Inferior results were observed when traditional Lewis acids such as $\mathrm{ZnCl}_{2}$ and $\mathrm{FeCl}_{3}$ were used. A progressive decrease in the ionic radii of $\mathrm{Ln}$ (III) from the light rare earth La to heavy rare earth $\mathrm{Yb}$ had a significant influence

Table 1 Condition screening for the reaction of $\mathbf{1}$ with $\mathbf{2} \mathbf{a}^{\text {a) }}$

\begin{tabular}{cccccc}
\hline Entry & $\begin{array}{c}\mathrm{Catalyst}_{(\mathrm{mol} \%)} \\
(\mathbf{1}: \mathbf{2 a})\end{array}$ & $\begin{array}{c}\text { Molar ratio } \\
(\mathrm{h})\end{array}$ & $\begin{array}{c}\text { Time } \\
\left({ }^{\circ} \mathrm{C}\right)\end{array}$ & $\begin{array}{c}\text { Yield } \\
(\%)\end{array}$ \\
\hline 1 & - & $1: 2$ & 4 & 50 & 0 \\
2 & $\mathrm{YbCl}_{3}(10)$ & $1: 2$ & 3 & 50 & 84 \\
3 & $\mathrm{YbCl}_{3}(10)$ & $1: 2$ & 4 & 50 & 99 \\
4 & $\mathrm{YbCl}_{3}(2.5)$ & $1: 2$ & 4 & 50 & 90 \\
5 & $\mathrm{YbCl}_{3}(1)$ & $1: 1.2$ & 4 & 50 & $99(99)^{\mathrm{b})}$ \\
6 & $\mathrm{YbCl}_{3}(2.5)$ & $1: 1$ & 4 & 50 & 95 \\
7 & $\mathrm{YbCl}_{3}(2.5)$ & $1: 1.2$ & 4 & 40 & 71 \\
8 & $\mathrm{YbCl}_{3}(2.5)$ & $1: 1.2$ & 4 & 50 & 95 \\
9 & $\mathrm{ErCl}_{3}(2.5)$ & $1: 1.2$ & 4 & 50 & 32 \\
10 & $\mathrm{GdCl}_{3}(2.5)$ & $1: 1.2$ & 4 & 50 & 24 \\
11 & $\mathrm{SmCl}_{3}(2.5)$ & $1: 1.2$ & 4 & 50 & 10 \\
12 & $\mathrm{LaCl}_{3}(2.5)$ & $1: 1.2$ & 4 & 50 & 33 \\
13 & $\mathrm{ZnCl}_{2}(2.5)$ & $1: 1.2$ & 4 & 50 & 6 \\
14 & $\mathrm{FeCl}_{3}(2.5)$ & 4 & 4 & 59 \\
\hline
\end{tabular}

a) Reactions were carried out under solvent-free conditions. b) The reaction was performed under an air atmosphere. on the reaction. The order of catalytic activity of these lanthanide ions is simply the reverse of their ionic radii. As a result, $\mathrm{YbCl}_{3}$ was chosen as a representative lanthanide source for carrying out the following studies.

To our delight, this Lewis acid-catalyzed reaction proceeded under mild, simple, and clean conditions. First, no solvent is required during the reaction, which not only negates the use of auxiliary reagents that may be toxic or flammable but also simplifies the operation. Second, the reaction works well under an air atmosphere (entry 6), so the steps of drying and protection, which are usually essential for most of the reactions involving lanthanide compounds, are unnecessary. As a result, this procedure is an efficient, convenient and environmentally friendly protocol for the synthesis of 2-amino-4H-3,1-benzothiazines.

The reaction scope of this $\mathrm{YbCl}_{3}$ catalytic system and its tolerance toward functional groups were explored using various isothiocyanates (Scheme 2). As shown in Table 2, all of the reactions with ortho-, meta- and para-substituted aryl isothiocyanates proceeded smoothly and afforded the expected products in good to excellent yields. Both electronic and steric effects were observed. The electron-donating group (EDG)-substituted aryl isothiocyanates (para-substituted, entries 8 and 9) required increased temperature $\left(70^{\circ} \mathrm{C}\right)$ to realize yields close to those containing electron-withdrawing substituents (entries 2-5). For example, $p$-chlorophenyl isothiocyanate $(\mathbf{2 c})$ reacted with $\mathbf{1}$ at $50^{\circ} \mathrm{C}$ leading to the corresponding product in $95 \%$ yield, whereas $p$-methoxyphenyl isothiocyanate (2i) achieved the same yield only when the reaction was carried out at $70^{\circ} \mathrm{C}$. Increased temperature was also necessary for ortho-substituted aryl isothiocyanates (entries 10 and 11) to achieve the desired yields, indicating an obvious steric effect. The reaction encountered some difficulties when alkyl isothiocyanates with different steric hindrance (entries 12 and 13) were used as substrates. Methyl isothiocyanate worked well at $70^{\circ} \mathrm{C}$, realizing a yield similar to those of EDG-substituted aryl isothiocyanates. However, sterically hindered cyclohexyl isothiocyanate gave a relatively lower yield despite extending the reaction time to $48 \mathrm{~h}$.

The high efficiency of the reaction compelled us to deduce a plausible mechanism. It was considered that the required 2-amino-4H-3,1-benzothiazine can be formed via a threestep sequence (Scheme 3). The first step of the reaction is the formation of thiourea $\mathbf{C}$, which can be detected and<smiles>[R]NC1=Nc2ccccc2C(N[R])=Nc2ccccc2C(CC(=O)OCC)S1</smiles> 
Table $2 \mathrm{YbCl}_{3}$-catalyzed reaction of $\mathbf{1}$ with various isothiocyanates ${ }^{\text {a) }}$

\begin{tabular}{|c|c|c|c|c|c|}
\hline Entry & $\mathrm{R}$ & Temp. $\left({ }^{\circ} \mathrm{C}\right)$ & Time (h) & Product & Yield (\%) \\
\hline 1 & $\mathrm{Ph}$ & 50 & 4 & $3 a+4 a$ & 99 \\
\hline 2 & $p-\mathrm{FC}_{6} \mathrm{H}_{4}$ & 50 & 4 & $3 b+4 b$ & 91 \\
\hline 3 & $p-\mathrm{ClC}_{6} \mathrm{H}_{4}$ & 50 & 4 & $3 c+4 c$ & 95 \\
\hline 4 & $p-\mathrm{BrC}_{6} \mathrm{H}_{4}$ & 50 & 4 & $3 d+4 d$ & 88 \\
\hline 5 & $p-\mathrm{NO}_{2} \mathrm{C}_{6} \mathrm{H}_{4}$ & 50 & 4 & $3 e+4 e$ & 91 \\
\hline 6 & $m-\mathrm{ClC}_{6} \mathrm{H}_{4}$ & 50 & 4 & $3 f+4 f$ & 99 \\
\hline 7 & $m-\mathrm{MeOC}_{6} \mathrm{H}_{4}$ & 50 & 4 & $3 g+4 g$ & 96 \\
\hline 8 & $p-\mathrm{MeC}_{6} \mathrm{H}_{4}$ & 70 & 4 & $3 h+4 h$ & 96 \\
\hline 9 & $p-\mathrm{MeOC}_{6} \mathrm{H}_{4}$ & 70 & 4 & $3 \mathbf{i}+4 \mathbf{i}$ & 95 \\
\hline 10 & $o-\mathrm{FC}_{6} \mathrm{H}_{4}$ & 70 & 4 & $3 \mathbf{j}+4 \mathbf{j}$ & 96 \\
\hline 11 & $o-\mathrm{ClC}_{6} \mathrm{H}_{4}$ & 70 & 4 & $3 k+4 k$ & 96 \\
\hline 12 & $c$-hexyl & 70 & 48 & $31+41$ & 74 \\
\hline 13 & $\mathrm{CH}_{3}$ & 70 & 4 & $3 m+4 m$ & $91(91)^{\mathrm{b})}$ \\
\hline
\end{tabular}

a) Typical reaction conditions: 1 : isothiocyanate $=1: 1.2,2.5 \mathrm{~mol} \% \mathrm{YbCl}_{3}$, solvent-free. b) The reaction was performed at $50^{\circ} \mathrm{C}$ for $15 \mathrm{~h}$.

isolated during the reaction, through the addition of the aromatic amino group to the isothiocyanate. Then, cyclization of $\mathbf{C}$ takes place via an intramolecular Michael addition of the S-terminal of thiourea to the lanthanide-activated $\alpha, \beta$ unsaturated ester, leading to intermediate D. Finally, an intramolecular hydride transfer forms the target 2-amino$4 H-3,1$-benzothiazine and releases the active $\mathrm{Ln}(\mathrm{III})$ catalyst.

\section{Conclusions}

In conclusion, an efficient method to synthesize 2-amino-



Scheme 3
4H-3,1-benzothiazines by $\mathrm{YbCl}_{3}$-catalyzed addition-cyclization reaction of $o$-aminocinnamate with isothiocyanates under solvent-free conditions has been developed. This tandem reaction is clean and can be handled easily. The environmentally friendly features of this catalytic procedure make it a practical and environmentally acceptable method for the synthesis of 2-amino-4H-3,1-benzothiazines.

This work was supported by the National Natural Science Foundation of China (20872106, 20972107) and the Priority Academic Program Development of Jiangsu Higher Education Institutions.

1 Dondoni A, Battaglia A, Giorgianni P. Cycloaddition of $N$-arylketenimines to thiobenzophenones. Formation of $4 \mathrm{H}$-3,1-benzothiazine and 2-iminothietan derivatives. J Chem Soc, Chem Commun, 1977, 43-44

2 Dondoni A, Battaglia A, Giorgianni P. Selectivity in ketenimine-thioketone cycloadditions. 1. 1,4- and 1,2-Addition pathways and the synthesis of 4H-3,1-benzothiazines, 2-iminothietanes, and thioacrylamides. J Org Chem, 1980, 45: 3766-3773

3 Nomura Y, Hayama T, Takeuchi Y, et al. The Willgerodt-Kindler reaction between polycyclic aromatic amines and benzaldehyde. Formation of 1,3-thiazines. Bull Chem Soc Jpn, 1984, 57: 1276-1278

4 Carisi P, Mazzanti G, Zani P, et al. Chemistry of silyl thioketones. Part 3. Cycloaddition reactions with heterodienes. J Chem Soc, Perkin Trans 1, 1987, 2647-2651

5 Nishio T. Reaction of $(1, \omega)-N$-acylamino alcohols with Lawesson's reagent: Synthesis of sulfur-containing heterocycles. J Org Chem, 1997, 62: 1106-1111

6 Nishio T, Sekiguchi H. Sulfur-containing heterocycles derived by reaction of $N$-thioacylamino alcohols with Lawesson's reagent and saponification of $\mathrm{N}$-thioacylamino esters. Heterocycles, 2002, 58: 203212

7 Csomós P, Fodor L, Bernáth G, et al. Novel $\beta$-lactam condensed 3-thiaquinolines: An efficient synthesis and structural characterization. Tetrahedron, 2008, 64: 1002-1011

8 Abaev V T, Tsiunchik F A, Gutnov A V, et al. Synthesis of 2,4-difuryl-4H-3,1-benzothiazines via a furan ring migration reaction. $\mathrm{J}$ Heterocyclic Chem, 2008, 45: 475-482 
9 Gimbert C, Vallribera A. A straightforward synthesis of benzothiazines. Org Lett, 2009, 11: 269-271

10 Hoechst A G. 3,1-Benzothiazine derivatives and process for their manufacture. GB1214000, 1970-11-25

11 Dreikorn B A. Tetrazolo- and triazolobenzothiazines. US Patent 4001227, 1975-12-22

12 El-Desoky S I, Kandeel E M, Abd-el-Rahman A H, et al. Synthesis and reactions of 4H-3,1-benzothiazines. J Heterocyclic Chem, 1999, 36: $153-160$

13 Matysiak J. Synthesis, antiproliferative and antifungal activities of some 2-(2,4-dihydroxyphenyl)-4H-3,1-benzothiazines. Bioorg Med Chem, 2006, 14: 2613-2619

14 Pelosi S S. 2-(3-Chloroanilino)-4H-3,1-benzothiazine. US 4002622, 1975-10-30

15 Su Y, Guo Q, Wang G, et al. 2-Imino-tetrahydro-1,3-benzothiazine derivative and its preparing method. CN 1683349, 2005-02-28

16 Rieu J P, Patoiseau J F, John G W, et al. Heterocyclic compounds for treating myocardial ischemia. US 6011032, 2000-1-4

17 Anzini M, Giordani A, Makovec F, et al. Amidine, thiourea and guanidine derivatives of 2-aminobenzothiazoles and aminobenzothiazines, novel pharmacological agents for the treatment of neurodegenerative pathologies. WO 2009/040331A2, 2009-4-2

18 Prieto J, Vega A, Moragues J. New heterocyclic derivatives of 1'and 3'-amino-5',6', 7', 8'-tetrahedro-2'-acetonaphthones. J Heterocyclic Chem, 1976, 13: 813-819

19 Gauthier J, Duceppe J S. Synthesis of novel imidazo[1,2-a][3,1] benzothiazines 4 , imidazo[1,2-a][1,2,4]benzotriazines 5 , and $4 \mathrm{H}$-imidazo[2,3-c]pyrido[2,3-e][1,4]oxazines 6. J Heterocyclic Chem, 1984, 21: $1081-1086$

20 Hari A, Miller B L. Rapid and efficient synthesis of 2-amino-4Hbenzothiazines. Org Lett, 2000, 2: 3667-3670

21 Fedotov A N, Trofimova E V, Sidorov V A, et al. 2-Amino-4H-3,1benzothiazines from arylcyclopropanes: crystal structure of the product of $\mathrm{N}^{1}$-(2-cyclopropylphenyl)- $\mathrm{N}^{2}$-(2-ethylphenyl)thiourea rearrangement. Doklady Chem, 2005, 405: 217-221

22 Fedotov A N, Trofimova E V, Romanov V A, et al. Synthesis of 2-amino-4H-3,1-benzoxazines and 2-amino-4H-3,1-benzothiazines by the rearrangement of $o$-cyclopropylphenylureas and $o$-cyclopropylphenylthioureas. Chem Heterocycl Comp, 2008, 44: 96-105

23 Ding Q, Wu J. A facile route to 2,4-dihydro- $1 H$-benzo $[d][1,3]$ thiazines via silver-catalyzed tandem addition-cyclization reactions. J Comb Chem, 2008, 10: 541-545

24 Tang R Y, Luo P S, Zhang X G, et al. Silver-catalyzed tandem ammonolysis-cyclization of 2-alkynylbenzenamines with tetraalkylthiuram disulfides to 4 -methylene-4H-benzo- $[d][1,3]$ thiazin-2-amines. Synlett, 2010, 1345-1350

25 Gonda J, Kristian P. Some nucleopidlic reactions of 2-isothiocyanatobenzyl bromide. A new simple synthesis of 2-substituted $4 \mathrm{H}$ benzo[d][1,3]-thiazines. Collection Czechoslovak Chem Commun, 1986, 51: 2802-2809

26 Schmittel M, Mahajan A, Steffen J P. A new facile synthesis of exomethylene 3,1-benzothiazines. Synthesis, 2004, 415-418

27 Sharma K K, Ralhan N K, Narang K S. Pyrimidobenzothiazine derivatives. II. The condensation of isothiocyano ketones and aryl amines. J Org Chem, 1963, 28: 740-742

28 Hull R, van den Broek P J, Swain M L. Reactions of heterocycles with thiophosgene. Part IV. $\beta, 4$-Dichloro-2-isothiocyanatocinnamaldehyde, a product from 4,7-dichloroquinoline. J Chem Soc, Perkin Trans 1, 1975, 922-925
29 Gonda J, Kristian P. 2-Isothiocyanatobenzylpyridinium bromide-An intermediate for the synthesis of 2-arylamino- $4 H$-benzo[d][1,3]thiazines. Collection Czechoslovak Chem Commun, 1986, 51: 2810-2816

30 Ding Q, Cao B, Zong Z, et al. Silica gel-promoted tandem additioncyclization reactions of 2-alkynylbenzenamines with isothiocyanates. J Comb Chem, 2010, 12: 370-373

31 Fukamachi S, Konishi H, Kobayashi K. Synthesis of 2-(2-dialkylamino-4H-3,1-benzothiazin-4-yl)acetic acid derivatives and 2-(2-thioxo1,2,3,4-tetrahydroquinazolin-4-yl)acetic acid derivatives. Synthesis, 2010, 1593-1598

32 Han X, Xu F, Luo Y, et al. An efficient one-pot synthesis of dihydropyrimidinones by a samarium diiodide catalyzed Biginelli reaction under solvent-free conditions. Eur J Org Chem, 2005, 15001503

33 Zhou Z, Xu F, Han X, et al. Stereoselective synthesis of pyrano [3,2-c]- and furano[3,2-c]quinolines: samarium diiodide-catalyzed one-pot aza-Diels-Alder reactions. Eur J Org Chem, 2007, 52655269

34 Wang J, Xu F, Cai T, et al. Addition of amines to nitriles catalyzed by ytterbium amides: An efficient one-step synthesis of monosubstituted $N$-arylamidines. Org Lett, 2008, 10: 445-448

35 Du Z, Li W, Zhu X, et al. Divalent lanthanide complexes: Highly active precatalysts for the addition of $\mathrm{N}-\mathrm{H}$ and $\mathrm{C}-\mathrm{H}$ bonds to carbodiimides. J Org Chem, 2008, 73: 8966-8972

36 Zhang H, Zhou Z, Yao Z, et al. Efficient synthesis of pyrimidinone derivatives by ytterbium chloride catalyzed Biginelli-type reaction under solvent-free conditions. Tetrahedron Lett, 2009, 50: 1622-1624

$37 \mathrm{Li} \mathrm{J}, \mathrm{Xu} \mathrm{F}$, Zhang Y, et al. Heterobimetallic lanthanide/sodium phenoxides: Efficient catalysts for amidation of aldehydes with amines. J Org Chem, 2009, 74: 2575-2577

38 Zhu X, Du Z, Xu F, et al. Ytterbium triflate: a highly active catalyst for addition of amines to carbodiimides to $N, N^{\prime}, N^{\prime \prime}$-trisubstituted guanidines. J Org Chem, 2009, 74: 6347-6349

39 Wang J, Li J, Xu F, et al. Anionic bridged bis(amidinate) lithium lanthanide complexes: Efficient bimetallic catalysts for mild amidation of aldehydes with amines. Adv Synth Catal, 2009, 351: 13631370

$40 \mathrm{Wu} \mathrm{Q}$, Zhou J, Yao Z, et al. Lanthanide amides $\left[\left(\mathrm{Me}_{3} \mathrm{Si}\right)_{2} \mathrm{~N}\right]_{3} \mathrm{Ln}(\mu-\mathrm{Cl})$ $\mathrm{Li}(\mathrm{THF})_{3}$ catalyzed hydrophosphonylation of aryl aldehydes. J Org Chem, 2010, 75: 7498-7501

41 Taylor M D, Carter C P. Preparation of anhydrous lanthanide halides, especially iodides. J Inorg Nucl Chem, 1962, 24: 387-391

42 CCDC 862963 contains the supplementary crystallographic data of 4a for this paper. These data can be obtained free of charge via http: //www.ccdc.cam.ac.uk/conts/retrieving.html

43 Allen F H, Kennard O, Watson D G, et al. Tables of bond lengths determined by x-ray and neutron diffraction. Part 1 . Bond lengths in organic compounds. J Chem Soc, Perkin Trans 2, 1987, S1-S19

44 Orpen A G, Brammer L, Allen F H, et al. Tables of bond lengths determined by $\mathrm{x}$-ray and neutron diffraction. Part 2. Organometallic compounds and coordination complexes of the d- and f-block metals. J Chem Soc, Dalton Trans, 1989, S1-S83

45 Molina P, Arques A, Molina A. A novel and efficient synthesis of 4H-3,1-benzoxazines by a tandem aza-Wittig/heterocumulene-mediated annulation strategy. Synthesis, 1991, 21-23

46 Garatt P J, Hobbs C J, Wrigglesworth R. Synthesis of 2-amino-4H-3,1benzoxazines from diphenyl cyanocarbonimidate. Facile replacement of the n-cyanoimine function by nitrogen nucleophiles. Tetrahedron, 1989, 45: 829-834

Open Access This article is distributed under the terms of the Creative Commons Attribution License which permits any use, distribution, and reproduction in any medium, provided the original author(s) and source are credited. 